\title{
The "hypertriglyceridemic waist" phenotype and glucose intolerance in pregnancy
}

\author{
Diane Brisson PhD, Patrice Perron MD MSc, Simon-Pierre Guay BSc, Daniel Gaudet MD PhD, \\ Luigi Bouchard PhD MBA
}

Previously published at www.cmaj.ca

ABSTRACT

Background: Abdominal visceral adiposity in early pregnancy has been associated with impaired glucose tolerance in later pregnancy. The "hypertriglyceridemic waist" phenotype (i.e., abdominal obesity in combination with hypertriglyceridemia) is a clinical marker of visceral obesity. Our study aimed to assess the association between the hypertriglyceridemic-waist phenotype in early pregnancy and glucose intolerance in later pregnancy.

Methods: Plasma triglycerides and waist girth were measured at 11-14 weeks of gestation among 144 white pregnant women. Glycemia was measured following a 75-g oral glucose tolerance test performed at 24-28 weeks of gestation.

Results: A waist girth greater than $85 \mathrm{~cm}$ in combination with a triglyceride level $\geq 1.7 \mathrm{mmol} / \mathrm{L}$ in the first trimester was associated with an increased risk of two-hour glucose $\geq 7.8 \mathrm{mmol} / \mathrm{L}$ following the $75-\mathrm{g}$ oral glucose tolerance test (odds ratio [OR] 6.1, $p=0.002$ ). This risk remained significant even after we controlled for maternal age, fasting glucose at first trimester and previous history of gestational diabetes (OR 4.7, $p=0.02$ ).

Interpretation: Measurement of waist girth in combination with measurement of triglyceride concentrations in the first trimester of pregnancy could improve early screening for gestational glucose intolerance.

$\mathrm{E}$ arly and accessible screening tools for gestational diabetes mellitus are needed to improve pregnancyrelated outcomes for women and children. ${ }^{1}$ Diagnostic tools currently used for gestational diabetes (most commonly the fasting oral glucose tolerance test) are expensive, time-consuming, uncomfortable for pregnant women and do not allow diagnosis before the end of the second trimester of pregnancy. ${ }^{2}$ Some earlier screening tools have been suggested, such as a 50-g glucose load followed by a one-hour plasma glucose analysis that can be performed at any time of the day and early in pregnancy. Although this test is less uncomfortable than the fasting oral glucose tolerance test, it remains time-consuming and unpleasant for women. Its use is therefore restricted mainly to women at risk for gestational diabetes.
Several attempts have been made to simplify these tests to promote wider use. However, no results have yet identified the means to carry out early and widely accessible screening. Interesting positive and negative predictive values have been obtained for first-trimester fasting glucose and insulin for subsequent gestational diabetes expression. ${ }^{3-5}$ However, these studies have been performed among women at risk of gestational diabetes and should be replicated in samples of women with various risk levels. Regardless of pregnancy, a person with normal fasting glucose can still meet the criteria for glucose intolerance or diabetes during an oral glucose tolerance test. Several studies have also shown that maternal prepregnancy obesity is directly associated with an increased risk of gestational diabetes. However, results vary widely across studies, possibly because of lack of specificity of tools for measurement of adiposity. ${ }^{6}$ A recent study has suggested that abdominal visceral adiposity, specifically, is associated with risk of gestational diabetes expression.

The hypertriglyceridemic-waist phenotype has been identified as a simple, easily available clinical marker of visceral obesity and related metabolic abnormalities. ${ }^{89}$ It is defined as the simultaneous presence of abdominal obesity (i.e., a waist girth greater than $85 \mathrm{~cm}$ in women or greater than $90 \mathrm{~cm}$ in men) and hypertriglyceridemia (i.e., a triglyceride concentration $\geq 2 \mathrm{mmol} / \mathrm{L}$ ). The aim of our study was to document the association between the presence of the hypertriglyceridemicwaist phenotype in early pregnancy and impaired glucose tolerance in later pregnancy.

\section{Methods}

\section{Participants and clinical data}

Our prospective cohort study comprised a sample of 144 French-Canadian white women with a singleton pregnancy who were each recruited at the beginning of their routine obstetric follow-up at Chicoutimi Hospital. All participants were sequentially recruited during their first visit, between

From the Department of Medicine, Université de Montréal, ECOGENE-21 and Lipid Clinic (Brisson, Perron, Guay, Gaudet, Bouchard), Chicoutimi Hospital, Saguenay, Que.; and the Department of Medicine (Perron), Université de Sherbrooke, Sherbrooke, Que.

CMAJ 2010. DOI:10.1503/cmaj.100378 
October 2006 and June 2009. We excluded women older than 40 years of age, women who had type 1 or type 2 diabetes or other disorders known to affect glucose metabolism before pregnancy, and women with a positive history of alcohol or drug abuse during their current pregnancy. Waist girth was determined at 11-14 weeks of gestation according to the standardized procedures of the Airlie conference. ${ }^{10}$ Measurement was performed at the mid-distance between the iliac crest and last rib margin while the woman was in a standing position, after normal expiration.

Written informed consent was obtained from all participants, and all clinical data were denominalized. The Chicoutimi Hospital Ethics Committee approved this project in accordance with the Declaration of Helsinki.

\section{Biochemical analysis}

Blood samples were obtained from the antecubital vein in the first trimester of pregnancy (i.e., 11-14 weeks of gestation) after a 12-hour overnight fast. Following centrifugation, plasma glucose concentrations were measured by the glucose oxidase method and plasma total triglyceride concentrations using enzymatic hydrolysis, on a Multiparity Analyzer CX7 (Beckman Instruments, Fullerton, USA). Plasma glucose concentrations were also measured at 0 and 120 minutes after a 12-hour fasting 75 -g oral glucose tolerance test performed at 24-28 weeks of gestation.

\section{Statistical analysis}

Participants were divided into four groups according to waist girth $(\leq 85$ or $>85 \mathrm{~cm})$ and triglyceride concentrations $(<1.7$ or $\geq 1.7 \mathrm{mmol} / \mathrm{L}$ ). The waist girth cut point of $85 \mathrm{~cm}$ was chosen based on the definition of the hypertriglyceridemic waist. ${ }^{8} \mathrm{How}-$ ever, considering the age of the participants and their relatively good metabolic condition, the more stringent recommended criteria of $1.7 \mathrm{mmol} / \mathrm{L}$ was chosen instead of $2 \mathrm{mmol} / \mathrm{L}$. Multivariate logistic regression models were built to calculate the relative odds of exhibiting a two-hour glucose level $\geq 7.8 \mathrm{mmol} / \mathrm{L}$ following the 75-g oral glucose tolerance test associated with these waist-triglyceride groups. The group characterized by both low waist girth and low triglyceride values was considered to be the reference group to which an odds ratio (OR) of one was set for comparison purposes. $P$ values were two-sided.

\section{Results}

Characteristics of participants are shown in Table 1. Of the 144 women included in this study, 19 had a previous history of gestational diabetes. Mean maternal age of participants was 28.3 (SD 3.9) years. At 11-14 weeks of gestation, their mean waist girth was 87.6 (SD 13.4) $\mathrm{cm}$, mean plasma triglyceride concentration was 1.16 (SD 0.53) $\mathrm{mmol} / \mathrm{L}$ and mean glycemia was 4.4 (SD 0.3) mmol/L. Almost all of them (143 of 144) had a normal fasting glucose level in their first trimester of pregnancy $(<5.3 \mathrm{mmol} / \mathrm{L})$. One woman had a fasting glucose level of $5.4 \mathrm{mmol} / \mathrm{L}$.

Abdominal obesity (waist $>85 \mathrm{~cm}$ ) in combination with hypertriglyceridemia (triglyceride concentration $\geq 1.7 \mathrm{mmol} / \mathrm{L}$ ) in the first trimester of pregnancy was associated with an increased risk of exhibiting a two-hour glucose level $\geq 7.8 \mathrm{mmol} / \mathrm{L}$ after the second trimester 75 -g oral glucose tolerance test (OR 6.1; $p=0.002$ ) (Table 2). This risk remained significant after we controlled for maternal age, fasting glucose at first trimester and previous history of gestational diabetes (OR $4.7 ; p=0.02$ ). The risk was not significantly increased among women with a waist circumference $>85 \mathrm{~cm}$ but with triglycerides $<1.7 \mathrm{mmol} / \mathrm{L}$ during the first trimester.

Notably, the trend in the odds of exhibiting a two-hour glucose $\geq 7.8 \mathrm{mmol} / \mathrm{L}$ remains the same when using cut points of $88 \mathrm{~cm}$ for waist circumference and $2 \mathrm{mmol} / \mathrm{L}$ for triglyceride levels. However, this analysis did not reach significance, probably because these more stringent criteria reduced the size of the last group (waist $>88 \mathrm{~cm}$ and triglyceride concentration $\geq 2.0 \mathrm{mmol} / \mathrm{L}$ ). The same trends were also observed using the criteria of the Canadian Diabetes Association for glucose intolerance (data not shown). Considering

Table 1: Characteristics of participants $(n=144)$ at the first trimester of pregnancy*

\begin{tabular}{|c|c|}
\hline Characteristic & No. $(\%)$ of woment \\
\hline \multicolumn{2}{|l|}{ Age, yr } \\
\hline Mean (SD) & $28.3(3.9)$ \\
\hline Median (range) & $28.0(18-38)$ \\
\hline \multicolumn{2}{|l|}{ Waist circumference, cm } \\
\hline Mean (SD) & $87.6(13.4)$ \\
\hline Median (range) & $86.0(62-164)$ \\
\hline \multicolumn{2}{|l|}{ Body mass index, $\mathrm{kg} / \mathrm{m}^{2}$} \\
\hline Mean (SD) & $24.7(4.6)$ \\
\hline Median (range) & $23.8(16.6-43.0)$ \\
\hline \multicolumn{2}{|l|}{ Total triglycerides, $\mathrm{mmol} / \mathrm{L}$} \\
\hline Mean (SD) & $1.16(0.53)$ \\
\hline Median (range) & $1.10(0.40-3.00)$ \\
\hline \multicolumn{2}{|l|}{ Fasting glycemia, $\mathrm{mmol} / \mathrm{L}$} \\
\hline Mean (SD) & $4.4(0.3)$ \\
\hline Median (range) & $4.4(3.4-5.4)$ \\
\hline Prior gestational diabetes & $19(13.2)$ \\
\hline Current smoker & 12 (8.3) \\
\hline \multicolumn{2}{|l|}{ Gravidity $\ddagger$} \\
\hline 0 & $56(38.9)$ \\
\hline 1 & $51(35.4)$ \\
\hline 2 & $27(18.8)$ \\
\hline 3 & $6 \quad(4.2)$ \\
\hline 4 & $4 \quad(2.8)$ \\
\hline \multicolumn{2}{|l|}{ Parity } \\
\hline 0 & $64(44.4)$ \\
\hline 1 & $62(43.1)$ \\
\hline 2 & $17(11.8)$ \\
\hline 3 & $1 \quad(0.7)$ \\
\hline
\end{tabular}

Note: SD = standard deviation.

*Measured at 11-14 weeks of gestation.

tUnless stated otherwise.

fExcluding the current pregnancy. 
their nonsignificant effect $(p>0.1)$, body mass index and family history of diabetes were not included in the multivariate model.

\section{Interpretation}

We found that the simultaneous presence of abdominal obesity and hypertriglyceridemia in the first trimester is associated with a significantly increased risk of glucose intolerance later in pregnancy. Gestational diabetes is a major public health concern and could lead to deleterious short- and longterm consequences for both women and their children. ${ }^{11,12}$ In response to the increasing prevalence of obesity and unfavourable lifestyle habits worldwide, gestational diabetes may be expected to become significantly more frequent in the next years. ${ }^{13}$ This trend will inevitably compel the development of early, clinically accessible means to screen for gestational diabetes in order to improve preventive strategies. ${ }^{1}$ Our results suggest that the hypertriglyceridemic-waist phenotype could be a practical early screening tool for gestational diabetes. Although some observations have suggested that first-trimester fasting hyperglycemia could also be a significant predictor of gestational diabetes expression, ${ }^{3,4}$ our results were observed among women who all had a normal first-trimester fasting glucose level.

The hypertriglyceridemic-waist phenotype is known as a simple, easily available marker of visceral obesity. ${ }^{8,9}$ Our results agree with those of Martin and colleagues, ${ }^{7}$ which showed the measurement of visceral adiposity in early pregnancy to be associated with risk for gestational glucose intolerance. However, visceral adiposity is measured using ultrasonography, which is not easily accessible, especially at the beginning of pregnancy when follow-up often takes place in small obstetrics and general practice clinics. Access to hospital technology such as ultrasonography comes only later during pregnancy, and usually not before the second trimester. This delayed access is particularly true in remote areas. In some isolated communities, this technology is simply not accessible, or trained staff is not available. Consequently, although Martin's results are very promising for early gestational diabetes screening, they are not easily transferable to current clinical practice. Our results have the advantage of suggesting an alter-

Table 2: Estimated risk for glycemia $\geq 7.8 \mathrm{mmol} / \mathrm{L}$ on a two-hour glucose tolerance test in the second trimester of pregnancy associated with expression of the hypertriglyceridemic-waist phenotype in the first trimester

\begin{tabular}{lcccc}
\hline $\begin{array}{l}\text { Waist, } \mathrm{cm} / \text { triglyceride } \\
\text { level, mmol/L }\end{array}$ & $\begin{array}{c}<.8 \mathrm{mmol} / \mathrm{L} \\
n=106\end{array}$ & $\begin{array}{c}\geq 7.8 \mathrm{mmol} / \mathrm{L} \\
n=38\end{array}$ & $\mathrm{OR}^{*}(95 \% \mathrm{Cl})$ & $\begin{array}{c}\text { Adjusted ORt } \\
(95 \% \mathrm{Cl})\end{array}$ \\
\hline$\leq 85 /<1.7$ & 54 & 10 & $1.0(\mathrm{ref})$ & $1.0(\mathrm{ref})$ \\
$\leq 85 / \geq 1.7$ & 4 & 2 & $2.7(0.4-16.8)$ & $4.2(0.6-27.5)$ \\
$>85 /<1.7$ & 40 & 17 & $2.3(0.95-5.5)$ & $2.1(0.8-5.5)$ \\
$>85 / \geq 1.7$ & 8 & 9 & $6.1(1.9-19.5)$ & $4.7(1.3-17.5)$ \\
\hline
\end{tabular}

Note: $\mathrm{Cl}=$ confidence interval, $\mathrm{OR}=$ odds ratio, ref = reference group

* No other variable was included in the model.

tThe model also included maternal age, fasting glucose at first trimester and history of gestational diabetes. native early screening tool for gestational glucose intolerance that is simple, readily accessible and inexpensive.?

\section{Strengths and limitations}

We chose the criteria of the World Health Organization to define gestational glucose intolerance on the basis of their worldwide use. As well, we set out to study metabolism by means of intermediate glucose intolerance instead of gestational diabetes. ${ }^{14,15}$ We believe this choice to be a strength of our study. A large cohort study found that women without gestational diabetes and only mildly elevated glucose concentrations during pregnancy had an increased risk of postpartum diabetes and may benefit from increased (and early) monitoring of their glucose metabolism during pregnancy.${ }^{16}$ Our results suggest a way to estimate individual risk to improve this kind of close monitoring.

One limitation of our study was our modest sample size. It did not allow for the various subdivisions (e.g., by parity) that would help to better document the usefulness of the hypertriglyceridemic-waist phenotype in the identification of women with a higher risk of gestational glucose intolerance or diabetes. Information obtained from previous pregnancies is very useful to evaluate the risk of glucose metabolism perturbations, or other gestational perturbations, during prenatal follow-up. This information is, of course, unavailable for primiparous women. Therefore, the replication of our results in a sample including only primiparous women would be even more important for this population. It would open the door to the development of an additional tool to help assess individual risks. In addition, a larger sample would allow for identification of optimal waist girth and plasma triglyceride concentration cut points. In the present study, we chose the cut point of $85 \mathrm{~cm}$ for waist girth based on the definition of the hypertriglyceridemic-waist phenotype proposed by Lemieux and colleagues, ${ }^{8,17}$ and we decided to use the more stringent recommended criteria of $1.7 \mathrm{mmol} / \mathrm{L} .{ }^{18}$ Our results should be replicated in larger samples and diversified populations in order to identify the optimal cut points.

Other limitations of our study were the ethnic homogeneity of participants and their follow-up in a single centre. However, we do not believe that this is a major concern for the generalization of our results, considering that the hypertriglyceridemicwaist phenotype on which our hypothesis was based has been shown to be a significant marker of visceral obesity and related glucose metabolism perturbations in various diversified populations., ${ }^{9,17,19}$

\section{Conclusion}

Measurement of waist girth in combination with triglyceride concentrations in the first trimester of pregnancy may be useful in the improvement of early screening for gestational diabetes. Our results have yet to be replicated in larger samples and diversified populations, or with various ranges of gestational glucose intolerance. Further research is needed to identify optimal waist 
girth and plasma triglyceride cut points for this population and to evaluate the hypertriglyceridemic-waist phenotype as a potential screening or diagnostic test.

This article has been peer reviewed.

Competing interest: None declared.

Contributors: Diane Brisson conceived the study design, analyzed and interpreted the data and wrote the manuscript. Patrice Perron and Daniel Gaudet contributed to the design of the study and revised the manuscript. SimonPierre Guay participated in data collection and revised the manuscript. Luigi Bouchard participated in the analysis and interpretation of the data and revised the manuscript. All of the authors approved the final version of the manuscript submitted for publication.

Acknowledgements: The authors acknowledge the contributions of Nadia Mior, Chantale Aubut, Marie-Pierre Rivard, Gladys Pontat and Jeannine Landry for their dedicated work in this study.

Daniel Gaudet is holder of the Canada Research Chair in preventive genetics and community genomics. Simon-Pierre Guay is supported by a Canadian Institutes of Health Research (CIHR) Frederick Banting and Charles Best Canada Graduate Scholarship: Master's Awards and by the Fonds de la recherche en santé du Québec (FRSQ).

Funding: This project was supported by ECOGENE-21, the CIHR team in community genetics (grant no. CTP-82941), the FRSQ and Diabète Québec.

\section{REFERENCES}

1. Reece EA, Leguizamón G, Wiznitzer A. Gestational diabetes: the need for a common ground. Lancet 2009;373:1789-97.

2. American Diabetes Association. Standards of medical care in diabetes - 2009 Diabetes Care 2009;32(Suppl 1):S13-61.

3. Riskin-Mashiah S, Younes G, Damti A, et al. First-trimester fasting hyperglycemia and adverse pregnancy outcomes. Diabetes Care 2009;32:1639-43.

4. Bitó T, Nyári T, Kovács L, et al. Oral glucose tolerance testing at gestational weeks or $=16$ could predict or exclude subsequent gestational diabetes mellitus during the current pregnancy in high risk group. Eur J Obstet Gynecol Reprod Biol 2005; 121:51-5

5. Bitó T, Földesi I, Nyári T, et al. Prediction of gestational diabetes mellitus in a high-risk group by insulin measurement in early pregnancy. Diabet Med 2005;22: 1434-9.

6. Torloni MR, Betrán AP, Horta BL, et al. Prepregnancy BMI and the risk of gestational diabetes: a systematic review of the literature with meta-analysis. Obes Rev 2009; 10:194-203.

7. Martin AM, Berger H, Nisenbaum R, et al. Abdominal visceral adiposity in the first trimester predicts glucose intolerance in later pregnancy. Diabetes Care 2009;32:1308-10

8. Lemieux I, Poirier P, Bergeron J, et al. Hypertriglyceridemic waist: A useful screening phenotype in preventive cardiology? Can J Cardiol 2007;23:23B-31B.

9. Sam S, Haffner S, Davidson MH, et al. Hypertriglyceridemic waist phenotype predicts increased visceral fat in subjects with type 2 diabetes. Diabetes Care 2009;32: 1916-20.

10. The Airlie (VA) Consensus Conference. In: Lohman T, Roche A, Martorel R, editors. Standardization of anthropometric measurements. Champaign (IL): Human Kinetics; 1988. p. 39-80.

11. Lim S, Choi SH, Park YJ, et al. Visceral fatness and insulin sensitivity in women with a previous history of gestational diabetes mellitus. Diabetes Care 2007;30:348-53.

12. Clausen TD, Mathiesen ER, Hansen T, et al. Overweight and the metabolic syndrome in adult offspring of women with diet-treated gestational diabetes mellitus or type 1 diabetes. J Clin Endocrinol Metab 2009;94:2464-70.

13. Ferrara A. Increasing prevalence of gestational diabetes mellitus. A public health perspective. Diabetes Care 2007;30:S141-6.

14. WHO Expert Committee on Diabetes Mellitus. World Health Organization. Technical report series. Geneva (Switzerland): The Organization; 1985.

15. World Health Organization. Definition, diagnosis and classification of diabetes mellitus and its complications. Geneva (Switzerland): The Organization; 1999.

16. Carr DB, Newton KM, Utzschneider KM, et al. Modestly elevated glucose levels during pregnancy are associated with a higher risk of future diabetes among women without gestational diabetes mellitus. Diabetes Care 2008;31:1037-9.

17. Lemieux I, Pascot A, Couillard C, et al. Hypertriglyceridemic waist: A marker of the atherogenic metabolic triad (hyperinsulinemia; hyperapolipoprotein B; small, dense LDL) in men? Circulation 2000;102:179-84.

18. McPherson R, Frohlich J, Fodor G, et al. Canadian cardiovascular society position statement-recommendations for the diagnosis and treatment of dyslipidemia and prevention of cardiovascular disease. Can J Cardiol 2006;22:913-27.

19. Gazi IF, Filippatos TD, Tsimihodimos V, et al. The hypertriglyceridemic waist phenotype is a predictor of elevated levels of small, dense LDL cholesterol. Lipids 2006;41:647-54

Correspondence to: Dr. D. Brisson, ECOGENE-21, Université de

Montréal, Chicoutimi Hospital, 225 St-Vallier, Chicoutimi QC

G7H 7P2; diane.brisson@ecogene21.org 